\title{
Design of influenza diagnostic instrument based on biochemiluminescence
}

\author{
Xuexiang $\operatorname{Lin}^{1,2}$, Zenggui Shen ${ }^{3 *}$ \\ ${ }^{1}$ Shenzhen Institutes of Advanced Technology, Chinese Academy of Sciences, 518055, China \\ ${ }^{2}$ University of Chinese Academy of Sciences, 518055, China \\ ${ }^{3}$ Department of Equipment, Nanfang Hospital, Southern Medical University, Guangzhou Guangdong 510515, China
}

\begin{abstract}
The design principle of a biochemiluminescent instrument is presented. The instrument diagnoses influenza based on detection of the flu viral neuraminidase activity. The instrument uses a photomultiplier tube (PMT) as sensor, with 24 test channels. The reaction disk is driven by step motor to rotate, where the reagent is incubated. Finally, PMT detects the number of photons emitted by the reagent and calculates the enzyme activity. The correlation coefficient $\left(\mathrm{R}^{2}\right)$ of 2.83 to $5.06 \log \mathrm{TCID}_{50} / \mathrm{ml}$ influenza virus was 0.9967 ; the detection limits of the two positive virus samples were $\mathrm{TCID}_{50} / \mathrm{mL}$ and $953 \mathrm{TCID}_{50} / \mathrm{mL}$, respectively, The positive predictive value (PPV) was $100 \%$, and the negative predictive value (NPV) was $98.90 \%$ in clinical evaluation. According to the evaluation, the designed biochemiluminescent instrument can well match with the reagents, and meet with the performance requirements of clinical setting.
\end{abstract}

\section{INTRODUCTION}

Influenza is caused by influenza virus infection. Influenza $\mathrm{A}, \mathrm{B}$, and $\mathrm{C}$ are 3 types of viruses responsible for illness, and type A is most likely to cause epidemic [1]. Influenza is mainly transmitted by air droplets. It often causes chills, headache, persistent cough, fatigue, nasal congestion and sore throat. In severe cases, it can cause pneumonia, myocarditis and heart failure.

The benefit of treatment is greatest when antiviral therapy is started within 24 hours of symptom onset, so a reliable diagnostic method enabled rapid test of influenza is essential ${ }^{[2][3]}$. At present, polymerase chain reaction (PCR) and colloidal gold are two main methods for influenza detection. PCR assay is based on the detection of flu viral gene sequences and colloidal gold is based on antigens, which are susceptible to genetic change of the viruses. In fact, new influenza viruses are constantly evolving by mutation or by reassortment, so a new detection method is needed for rapid diagnosis ${ }^{[4]}$.

Neuraminidase is one of the essential enzymes of influenza virus, which help progeny influenza virus cleave sialic acid and release from the host cell. Neuraminidase also contributes to virus binding to the sialic acid groups of cell glycoproteins, which could complement the receptor-binding function of hemagglutinin, enhancing enzymatic activities of neuraminidase, and facilitate virus infection ${ }^{[2]}$. Therefore, neuraminidase plays an important role in virus replication and transmission ${ }^{[5][6]}$. All type A and B influenza viruses carry neuraminidase, so neuraminidase can be an ideal marker for diagnosis of influenza ${ }^{[7][8]}$.
An assay method, the homogeneous biochemiluminescent assay (HBA) for rapid detection of influenza by detecting viral neuraminidase activity, has been developed [9][10]. It uses a luciferase-based biochemiluminescent substrate ${ }^{[11]}$. In the presence of influenza virus in a reaction, the substrate is cleaved to free luciferin, which becomes an active substrate of firefly luciferase. In the presence of firefly luciferase, the free luciferase is oxidized to oxyluciferin, resulting in a stable light signal that can be detected by instrument ${ }^{[12][13]}$. In this paper, we introduce the implementation of a HBA instrument for rapid influenza test based on viral neuraminidase activity.

\section{METHODOLOGY}

The whole instrument consists of reaction disk, heating module, measuring module, moving and supporting structure, as shown in Figure 1. The reaction disk has 24 test channels, which is the place for reaction mix incubation, reaction and luminescence. The reaction disk is fixed on a step motor. The motor moves to bring the reaction mix to the detection position, and the PMT is directly below the detection position, which is used to measure the light signal emitted by the reagent. The heating module is located at the inner side of the reaction disk, and pasted with a heating film and a temperature sensor. When the instrument works, the heating film and the temperature sensor works together to provide a constant temperature environment for the reaction disk. The instrument is equipped with a shield to prevent stray

\footnotetext{
* Corresponding author: 15297818386@163.com
} 
light from entering into the instrument and improve the performance of the instrument.

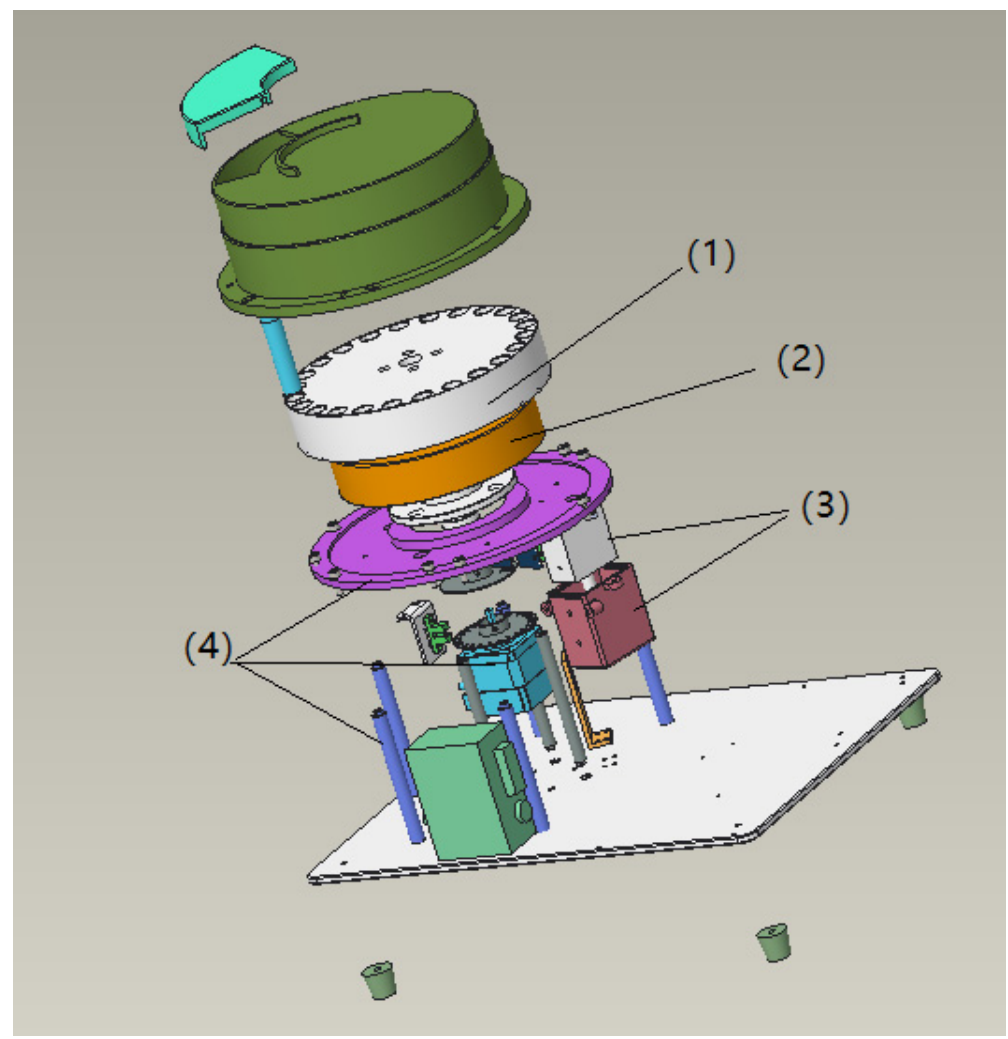

Fig.1.Instrument structure diagram. (1) Reaction disk; (2) heating module; (3) measuring module; (4) moving and supporting structure.

The results of the instrument are read as Relative Light Unit(RLU).A study was conducted to calculate the appropriate cut-off value. We tested 460 clinical samples, and the samples were cultured for virus as well. The
RLU results and virus culture results are listed in the table1. With the raw data, the true positive rate (TPR) and false positive rate (FPR) were calculated under different cut-off values.

Table1. The TPR and FPR under different cut-off value

\begin{tabular}{|c|c|c|c|c|c|c|c|}
\hline \multirow{2}{*}{$\begin{array}{l}\text { Cut-off } \\
\text { (k RLU) }\end{array}$} & Cultured & $\mathrm{P}$ & $\mathrm{P}$ & $\mathrm{N}$ & $\mathrm{N}$ & \multirow{2}{*}{ TPR (\%) } & \multirow{2}{*}{ FPR (\%) } \\
\hline & Test & $P$ & $\mathrm{~N}$ & $P$ & $\mathrm{~N}$ & & \\
\hline 50 & & 39 & 14 & 152 & 255 & 73.58 & 37.35 \\
\hline 100 & & 37 & 16 & 79 & 328 & 69.81 & 19.41 \\
\hline 180 & & 36 & 17 & 46 & 361 & 67.92 & 11.30 \\
\hline 190 & & 35 & 18 & 43 & 364 & 66.04 & 10.57 \\
\hline 200 & & 35 & 18 & 36 & 371 & 66.04 & 8.85 \\
\hline 210 & & 35 & 18 & 35 & 372 & 66.04 & 8.60 \\
\hline 220 & & 35 & 18 & 32 & 375 & 66.04 & 7.86 \\
\hline 230 & & 34 & 19 & 31 & 376 & 64.15 & 7.62 \\
\hline 240 & & 34 & 19 & 28 & 379 & 64.15 & 6.88 \\
\hline 300 & & 33 & 20 & 14 & 393 & 62.26 & 3.44 \\
\hline 400 & & 33 & 20 & 9 & 398 & 62.26 & 2.21 \\
\hline 500 & & 31 & 22 & 3 & 404 & 58.49 & 0.74 \\
\hline 600 & & 31 & 22 & 0 & 407 & 58.49 & 0.00 \\
\hline 700 & & 30 & 23 & 0 & 407 & 56.60 & 0.00 \\
\hline 800 & & 29 & 24 & 0 & 407 & 54.72 & 0.00 \\
\hline 900 & & 28 & 25 & 0 & 407 & 52.83 & 0.00 \\
\hline 1,000 & & 26 & 27 & 0 & 407 & 49.06 & 0.00 \\
\hline 1,100 & & 26 & 27 & 0 & 407 & 49.06 & 0.00 \\
\hline 1,200 & & 26 & 27 & 0 & 407 & 49.06 & 0.00 \\
\hline 1,300 & & 26 & 27 & 0 & 407 & 49.06 & 0.00 \\
\hline
\end{tabular}




\begin{tabular}{|c|c|c|c|c|c|c|c|}
\hline \multirow{2}{*}{$\begin{array}{c}\text { Cut-off } \\
(\mathrm{k} \text { RLU) }\end{array}$} & Cultured & $\mathrm{P}$ & $\mathrm{P}$ & $\mathrm{N}$ & $\mathrm{N}$ & & \\
\cline { 2 - 6 } & Test & $\mathrm{P}$ & $\mathrm{N}$ & $\mathrm{P}$ & $\mathrm{N}$ & & \multirow{2}{*}{ FPR (\%) } \\
\hline 1,400 & & 26 & 27 & 0 & 407 & 49.06 & 0.00 \\
\hline 1,500 & & 25 & 28 & 0 & 407 & 47.17 & 0.00 \\
\hline
\end{tabular}

460 clinical samples were tested. TPR, FPR were calculated under different cut-off value. Gold standard method was virus culture. P: Positive; N: Negative. P: Positive; N: Negative.
The ROC curve is shown in Figure 2. Since the instrument focuses on clinical screening, in this study, $220 \mathrm{~K}$ (220000 RLU) was used as the cut-off value. The TPR, FPR were $66.04 \%, 7.86 \%$, respectively.

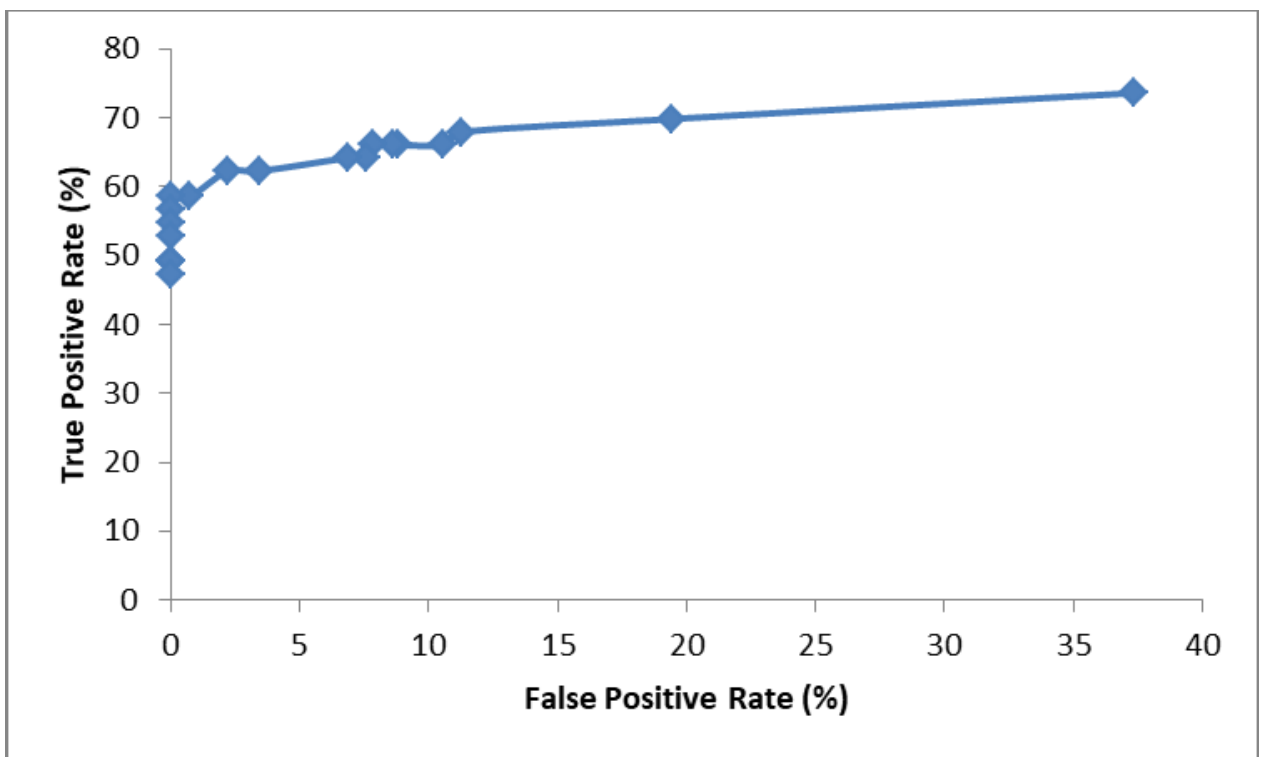

Fig. 2. The ROC curve of 460 clinical samples. Cut-off value ranges from $50 \mathrm{~K}$ to $1500 \mathrm{~K}$ RLU. TPR (\%) was plotted against FPR (\%).

with concentrations of 2.83 to $5.06 \log$ TCID50 $/ \mathrm{ml}$. The correlation coefficient (R2) was 0.9967 (95\% confidence interval: 0.9690-1.0; Figure 3)

\section{RESULTS}

To evaluate linearity and linear range, we tested samples of influenza virus strains (A/CA/07/2009; wild type)

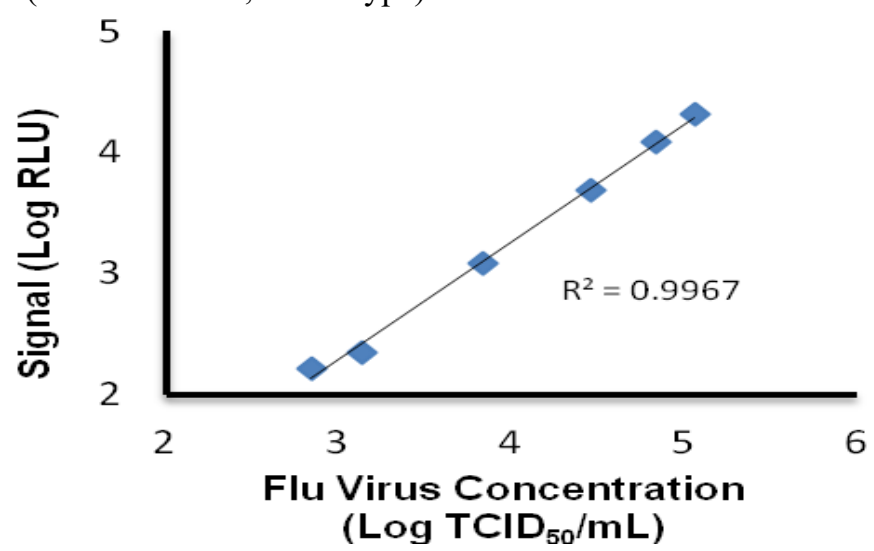

Fig. 3. Linearity and linear range of various influenza virus concentrations. Signal intensity (log RLU) was plotted against the flu virus concentration $(\log$ TCID50 /ml).

To evaluate the variability, two positive samples were tested every day, and each sample was repeated twice for 12 consecutive days. The results are shown as
Table2. The test of all samples (95\% confidence interval: $92.75 \%-99.95 \%$ ) can correctly diagnose whether there is influenza virus in the samples.

Table 2 . Repeatability of different samples

\begin{tabular}{|c|c|c|c|c|c|c|}
\hline & \multicolumn{3}{|c|}{ Positive } & \multicolumn{2}{c|}{$\begin{array}{c}\text { Positive } \\
\text { Sample 2 }\end{array}$} & $\begin{array}{c}\text { Negative } \\
\text { Sample }\end{array}$ \\
\cline { 2 - 7 } & 1 & 2 & 3 & 4 & 5 & 6 \\
\hline Mean RLU(n=48) & 373 & 1009 & 3933 & 724 & 3357 & 101 \\
\hline SD & 82 & 198 & 1003 & 228 & 352 & 34 \\
\hline
\end{tabular}




\begin{tabular}{|c|c|c|c|c|c|c|}
\hline \%CV & 21.91 & 19.68 & 25.50 & 31.49 & 10.48 & 33.59 \\
\hline \% Positive & 100 & 100 & 100 & 100 & 100 & 0 \\
\hline
\end{tabular}

To evaluate the limit of detection (LOD), we tested 5 samples of influenza virus strains A/CA/07/2009 and $\mathrm{A} / \mathrm{NC} / 37 / 2009$ for 20 times. The concentrations of the samples were close to the detection limit. The results are shown in Table 3. The diagnostic accuracy of positive

influenza samples should be at least $95 \%$, so the detection limits of $\mathrm{A} / \mathrm{CA} / 07 / 2009$ and $\mathrm{A} / \mathrm{NC} / 37 / 2009$ were 995 TCID $50 / \mathrm{mL}$ and 953 TCID $50 / \mathrm{mL}$, respectively.

Table 3.LOD at various concentrations of influenza virus strains

\begin{tabular}{|c|c|c|c|c|c|}
\hline \multirow{2}{*}{} & \multicolumn{3}{|c|}{$\mathrm{A} / \mathrm{CA} / 07 / 2009$} & \multicolumn{3}{c|}{$\mathrm{A} / \mathrm{NC} / 39 / 2009$} \\
\cline { 2 - 6 } & 1 & 2 & 3 & 1 & 2 \\
\hline Concentrations (TCID50/mL) & 663 & 995 & 1,326 & 953 & 1,271 \\
\hline Mean(S/CO) & 0.98 & 1.58 & 1.96 & 1.15 & 1.87 \\
\hline \% CV(S/CO) & 13.81 & 7.98 & 6.59 & 8.36 & 3.45 \\
\hline \% Positive & $45 \%$ & $100 \%$ & $100 \%$ & $100 \%$ & $100 \%$ \\
\hline
\end{tabular}

To evaluate the performance of the assay in clinical settings, a clinical study was designed and conducted. The study was approved by the medical ethics committee at Jiangmen Wuyi Hospital of Chinese traditional medicine, and conducted according to the requirements of the China Food and Drug Administration (CFDA).
From $25 / 01 / 2019$ to $22 / 07 / 2019,361$ participants were enrolled in the study. The results are presented in Table4. The positive predictive value (PPV) was $100 \%$, and the negative predictive value (NPV) was $98.90 \%$. The colloidal gold reagent of Guangzhou Wondfo Biotech Co., Ltd., was used as reference reagent.

Table 4. The test results from clinical study.

\begin{tabular}{|c|c|c|c|c|}
\hline \multicolumn{2}{|c|}{} & \multicolumn{2}{|c|}{$\begin{array}{c}\text { Results confirmed } \\
\text { by colloidal gold }\end{array}$} & \multirow{2}{*}{ total } \\
\cline { 2 - 5 } \multicolumn{2}{|c|}{} & Positive & 3 & 93 \\
\hline \multirow{2}{*}{$\begin{array}{c}\text { Results confirmed } \\
\text { by this assay }\end{array}$} & Negative & 90 & 268 & 268 \\
\cline { 2 - 5 } & & 90 & 271 & 361 \\
\hline
\end{tabular}

Use nasopharyngeal swab to collect sample. Swab should go down to nasopharynx to collect adequate sample. Tenderly rotate the swab 5 rounds. To ensure adequate sample collection, repeat the same operation in the other cavity. The samples were tested at the end of each day. The test results were recorded and saved according to relevant guidelines and regulations.

\section{CONCLUSION}

High mutation rate and large number of variants make the diagnosis of influenza a difficult problem. Neuraminidase is a conserved enzyme of influenza virus, which is not susceptible to virus mutation; therefore, it is an ideal influenza diagnostic marker. The instrument described in this paper is based on a luciferase-based biochemiluminescence assay, and can diagnose influenza by detecting neuraminidase. The instrument has no liquid circuit design, so it has the advantages of simple structure, high stability and low cost [14]. Experimental results show that the instrument and reagent can be suitable for use in POCT settings.

\section{References}

1. Eduardo Forleo-Neto, Elisa Halker, Verônica Jorge Santos, Terezinha Maria Paiva, João niolo-Neto.
Influenza. Rev Soc Bras Med Trop. 36(2):26774(2003).

2. Feng Wen,Xiu-Feng Wan. Influenza Neuraminidase: Underrated Role in Receptor Binding. Trends Microbiol.27(6):477-479(2019).

3. David Y Gaitonde, Faith C Moore, Mackenzie K Morgan. Influenza: Diagnosis and Treatment. 100(12):751-758(2019).

4. Robert G Webster, Elena A Govorkova. Continuing challenges in influenza. Ann N Y Acad Sci . 1323(1):115-39(2014)

5. Gillian M Air. Influenza neuraminidase. Influenza Other Respir Viruses. 6(4):245-56(2012).

6. Matthew J Sylte, David L Suarez. Influenza neuraminidase as a vaccine antigen. Curr Top Microbiol Immunol. 333:227-41(2009).

7. Zhizeng Gao, Masahiro Niikura, Stephen G Withers. Ultrasensitive Fluorogenic Reagents for Neuraminidase Titration. Angew Chem Int Ed Engl. 56(22):6112-6116(2017).

8. Zuzana Hlasová, Ivan Košík, Miroslav Ondrejovič, Stanislav Miertuš, Jaroslav Katrlík . Methods and Current Trends in Determination of Neuraminidase Activity and Evaluation of Neuraminidase Inhibitors. Crit Rev Anal Chem. 49(4):350-367(2019). 
9. Kwok Min Hui ,Xiao Jing Li,Lu Pan ,X. James Li. Conference on Advances in Global Health through Sensing Technologies. Cellex, Inc., 104 TW Alexander Drive Building 20, Research Triangle Park, NC USA 27709.94900N.1-94900N.6.( 2015).

10. Shengjun $\mathrm{Wu}, X u e x i a n g$ Lin,Kwok Min Hui,Su Yang,Xuanlan Wu,Yichen Tan,Meimei Li,Ai-Qing Qin,Qingxi Wang,Qi Zhao,Pengfei Ding,Kaisheng Shi,X James Li.A Biochemiluminescent Sialidase Assay for Diagnosis of Bacterial Vaginosis.Sci Rep.9(1):20024(2019).

11. $\mathrm{Y}$ Isojima, $\mathrm{T}$ Isoshima,K Nagai,K Kikuchi,H Nakagawa.Ultraweak biochemiluminescence detected from rat hippocampal slices.Neuroreport.6(4):658-60(1995).

12. Frank R Wettey, Antony $\mathrm{P}$ Jackson. Luciferase reporter assay. Subcell Biochem. 40:423-5(2006).

13. Roohullah Hemmati, Saman Hosseinkhani, Reza H Sajedi, Taha Azad, Amin Tashakor, Nuredin Bakhtiari , Farangis Ataei. Luciferin-Regenerating Enzyme Mediates Firefly Luciferase Activation Through Direct Effects of D-Cysteine on Luciferase Structure and Activity. Photochem Photobiol. 91(4):828-36(2015).

14. D Champiat, $\mathrm{N}$ Matas, $\mathrm{B}$ Monfort,H Fraass. Applications of biochemiluminescence to HACCP. Luminescence . 16(2):193-8(2001). 